\title{
Newly Developed Endotriptor for Patients with Basket Impaction in the Distal Common Bile Duct
}

We have developed a new endotriptor that can be used through the instrument channel of the duodenoscope. The coiled metal sheaths are $53 \mathrm{~cm}$ long and $2.4 \mathrm{~mm}$ in diameter (Figure $\mathbf{1} \mathbf{a}$ ), and fit over the remaining wire of the basket catheter. "Remaining wire" here means the length of wire between the entrance of the instrument channel port on the duodenoscope and the cut end of the wire. The sheaths are designed to be slightly shorter than the "remaining wire". This allows easy insertion of a sheath over the wire and into the endoscope. An Olympus JF200 duodenoscope and an FG 22-Q-1 or 403Q basket catheter (Olympus, Tokyo, Japan), the length and diameter of which are suitable for the new endotriptor, were used.

When basket impaction occurs with a bile stone in the distal common bile duct, the basket catheter is cut at the handle. Only the tube sheath should then be pulled out, leaving the wire inside the endoscope. Three coiled metal sheaths from the endotriptor are inserted over the basket wire and into the duodenoscope. The end of the sheath is then gripped with a vise (Figure $\mathbf{1}$ b). The endotriptor can be pulled out with the basket catheter after the stone has been crushed. In 20 patients who had basket impaction after endoscopic sphincterotomy and underwent lithotripsy with the new endotriptor, the stones were crushed successfully in all cases. There were no complications such as biliary perforation or bleeding.

The endotriptors currently available cannot be used without withdrawing the duodenoscope from the patient [1]. The duodenoscope then has to be reinserted to extract the fragments of a stone immediately after traditional lithotripsy. With the new endotriptor, the required maneuvers can be carried out without removing the duodenoscope - a considerable advantage. There is no chance of injuring the mucosa of the upper gastrointestinal tract with the new endotriptor, as it passes through the endoscope. In addition,

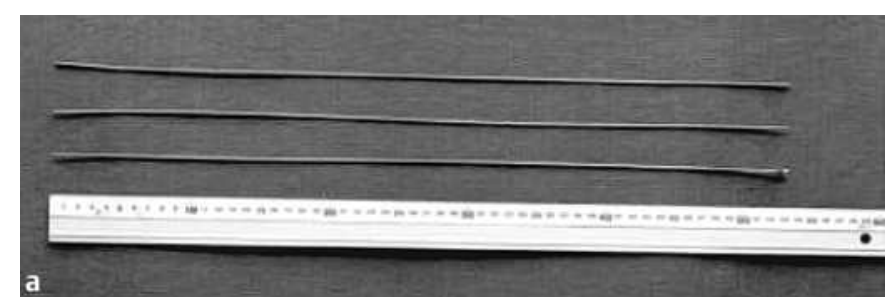

Figure 1 a The newly developed endotriptor consists of three coiled metal sheaths $53 \mathrm{~cm}$ long and $2.4 \mathrm{~mm}$ in diameter. $\mathbf{b}$ The end of the endotriptor is gripped in a vise.

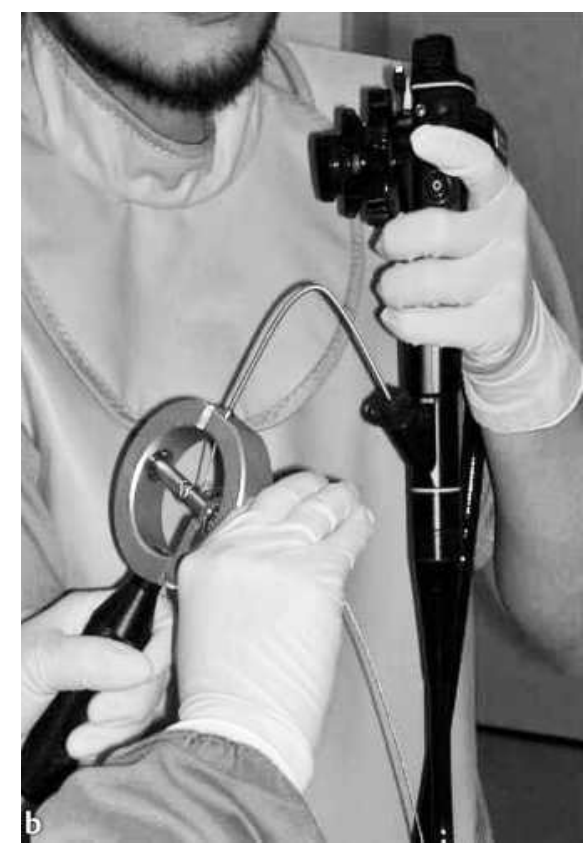

References

${ }^{1}$ Binmoeller KF, Bruckner M, Thonke F et al. Treatment of difficult bile duct stones using mechanical, electrohydraulic and extracorporeal shock wave lithotripsy. Endoscopy 1993; 25: $201-206$

Corresponding Author

\section{S. Kuriyama, M.D.}

Third Dept. of Internal Medicine Kagawa University School of Medicine 1750-1 Ikenobe

Miki-cho, Kita-gun

Kagawa 761-0793

Japan

Fax: $\quad$ +81-87-891-2158

E-mail: skuriyam@med.kagawa-u.ac.jp the distal common bile duct is never at risk of being perforated by the tip of the new endotriptor, as the impacted basket can be returned to the upper bile duct by pushing the endotriptor.

\section{T. Nakatsu1, N. Uchida², M. Ogawa ${ }^{2}$,}

\section{A. Muramatsu' ${ }^{1}$, A. Morishita ${ }^{1}$,}

H. Yoneyama ${ }^{1}$, K. Nomura ${ }^{1}$, T. Sokabe ${ }^{1}$,

T. Masaki², S. Kuriyama ${ }^{2}$

${ }^{1}$ Dept. of Gastroenterology, St. Martin

Hospital, Kagawa, Japan

${ }^{2}$ Third Dept. of Internal Medicine, Kagawa University School of Medicine, Kagawa, Japan. 